\title{
Interaction between the BDNF gene Val/66/Met polymorphism and morning cortisol levels as a predictor of depression in adult women
}

\author{
J. Herbert, M. Ban, G. W. Brown, T. O. Harris, A. Ogilvie, R. Uher and T. K. J. Craig
}

\section{Background}

Common genetic variants, such as the brain-derived neurotrophic factor (BDNF) Val/66/Met polymorphism (rs6265), are known to interact with environmental factors such as early adversity to increase the risk of subsequent major depression. Much less is known about how they interact with individual differences in cortisol, although these also represent a risk for major depression.

\section{Aims}

To determine whether this BDNF variant moderated the risk represented by higher levels of morning salivary cortisol in adult women.

\section{Method}

We recruited 279 premenopausal women who were at high risk of major depressive disorder because of either negative self-evaluation, unsupportive core relationship or chronic subclinical symptoms of depression or anxiety. Morning salivary cortisol was measured daily for up to 10 days at entry. Participants were followed up for about 12 months by telephone calls at 3-4 monthly intervals. Major depression and severe life events were assessed through interviews at baseline and follow-up; DNA was obtained from the saliva.

\section{Results}

There were 53 onsets (19\%) of depressive episodes during follow-up. There was a significant U-shaped relationship between adjusted morning cortisol levels at baseline and the probability of depression onset during follow-up. In total, 51\% experienced at least one severe life event/difficulty, and this strongly predicted subsequent onsets of depressive episodes. The BDNF Val/66/Met genotype was not directly associated with onsets of depression or with cortisol levels, but there was significant interaction between Val/66/Met and cortisol: the association between baseline cortisol and depression was limited to those with the Val/66/Val variant. There was no interaction between life events and either this BDNF polymorphism or cortisol levels.

\section{Conclusions}

Morning salivary cortisol interacts with the BDNF Val/66/Met polymorphism in predicting new depressive episodes. This paper adds to the evidence that single gene polymorphisms interact with endogenous factors to predict depression.

\section{Declaration of interest}

None.
Increasing evidence implicates brain-derived neurotrophic factor (BDNF) in depression. ${ }^{1}$ Brain-derived neurotrophic factor is essential for the developing brain, and plays a major role in plasticity of the adult brain. ${ }^{2,3}$ Antidepressant drugs, such as fluoxetine, increase levels of BDNF mRNA in the hippocampus. ${ }^{4-7}$ Blocking BDNF also prevents their action on rodent behaviours in experimental 'models' of depression. ${ }^{8}$ The same drugs stimulate mitotic activity of the progenitor cells in the adult hippocampus of the rat, and this also depends on BDNF. ${ }^{9,10}$ The parallels between factors that alleviate depression and those that increase hippocampal neurogenesis have led to the suggestion that the two are linked. ${ }^{11,12}$

Plasma levels of BDNF are lowered in depression, and increase with recovery, although how blood levels of BDNF relate to those in the brain is uncertain. ${ }^{13-15}$ Since depression is a frequent and moderately heritable disorder, common genetic variants are likely to contribute to its pathogenesis. A common variant at the Val/66/Met locus (rs6265) of the BDNF gene with the minor Met allele carried by $30 \%$ humans is associated with reduced activity-dependent secretion of BDNF, and altered processing of pro-BDNF to the mature molecule. ${ }^{16}$ Attempts to show a simple association between one or other BDNF variants and the occurrence of depression have not been consistent. ${ }^{17-19}$ A more promising approach has followed the recognition that genetic variants (for example in the serotonin transporter) may interact with elements in the environment, such as early or more proximal adversity, to determine the risk for depression, ${ }^{20}$ although some aspects of such studies remain controversial. ${ }^{21-24} \mathrm{~A}$ similar approach has been adopted with the BDNF Val/66/Met variant; two studies have reported an interaction between the Met allele and life events in predicting major depressive disorder. ${ }^{25,26}$ The association between adversity (maltreatment) during early life and subsequent depression is accentuated in those with the Met allele. $^{27-29}$

A less explored avenue is whether genetic variants may also interact with endogenous factors, such as cortisol. Damped diurnal rhythms of cortisol are well-established to occur in a proportion of cases of major depression, ${ }^{30}$ as does resistance to the normal negative feedback effect of administered glucocorticoids. ${ }^{31,32}$ Furthermore, both adolescents and adult women with levels of cortisol in the morning in the upper normal range are at higher risk of subsequent depression. ${ }^{33,34}$ Levels of morning salivary cortisol have been reported to interact with the BDNF Val/66/Val genotype (not the Met allele) to predict first onset depression in adolescents. ${ }^{35}$ A three-way association between life events, this BDNF variant and cortisol levels has not been found so far. The hypothesis explored in this paper is whether the Val or Met allele of the BDNF Val/66/Met variant is associated with morning cortisol levels as a predictor of the onset of a depressive episode in adult women; and, in particular, whether there is an interaction between the two measures. We examined three samples of participants, collected in the same way and according to the same criteria, but separated both by time of sampling and by domicile. In this way, we hoped to establish a more general pattern of risk in adult women than is usually the case. 


\section{Method}

\section{Samples}

The study population consisted of three samples. The participants in each sample were recruited in the same way, assessed at entry in a similar manner (with some minor differences, described below) and followed up in the same way for 12-18 months. Participants were premenopausal (except that sample 1 included women aged up to 66) were of European (White) descent and had at least one child. The levels of cortisol and its association with the prospective onset of major depressive disorder (but not the interaction with BDNF Val/66/Met) in sample 1 have already been reported. ${ }^{33}$

\section{Recruitment}

The location and date of each sample was as follows.

(a) Sample 1: Islington, Inner City of North London, 1996-1999, $n=116$, of whom 44 consented in 2004 to give further saliva for genotyping for this analysis.

(b) Sample 2: Southwark \& Lambeth, Inner City of South London, 2003-2006, $n=155$.

(c) Sample 3: Oxford, 2003-2006, $n=80$.

General practitioners in each area agreed to circulate all women in the relevant age group registered with their practice with a questionnaire as a preliminary to participating in some research about the health of women and their circumstances. Depression was not explicitly mentioned. They explained that they thought the research was worthwhile and hoped the recipients would find time to fill in the form and return it in a stamped addressed/freepost envelope to the research team's address giving their contact details if they were willing to participate further. Across the three areas some 7000 such forms were mailed out, of which 2388 were returned, in line with previous surveys of primary care lists, which often contain $30 \%$ names of persons who have moved on. The form contained information about current mental state, self-esteem and core relationship quality that would allow the identification of women who were not currently experiencing clinical depression but who were considered vulnerable through at least one of these three characteristics found from previous work to predict onset within the short term:

(a) negative evaluation of self

(b) negative elements in core relationships

(c) chronic subclinical conditions of depression or anxiety.

Eligible women $(n=523)$ were then telephoned to confirm eligibility. Women found to be vulnerable but, not currently depressed or receiving corticoid medication, but with adequate command of English were invited to take part. The procedures involved (which included providing morning saliva samples and tape-recorded interviews) were explained and a time arranged for baseline interview in the individual's house (or - if she preferred - at the research team's office). This identified 160 women not meeting inclusion criteria, 84 unwilling to take part and 279 eligible women willing to participate.

\section{Psychological measures}

At entry, participants were assessed with psychological and psychiatric instruments, including the following. The Schedule for Clinical Assessment in Neuropsychiatry (SCAN) ${ }^{36}$ was used to establish current mental state. Telephone screening had already excluded women with current clinical (or case-level) depression, but classifying minor symptoms was necessary for establishing the chronic 'subclinical' mood disorder component of the threefold vulnerability index. ${ }^{37}$ This component is referred to here as 'chronic subclinical conditions' of either depression, anxiety or obsessional disorder, chronic in the sense of having lasted continuously for at least 1 year. ${ }^{38}$ When the condition comprised anxiety rather than depressed mood it could involve disorder at a clinical as well as a subclinical level. Case-level depression was defined as either major depression as specified by DSM-IV, ${ }^{39}$ or Bedford College case-level depression. ${ }^{40}$ The latter is based on an algorithm derived from data on women, which can be approximated by following a checklist. This comprises a minimum of 2 weeks of depressed mood and at least four other very slightly different key symptoms (key symptoms in the DSM-IV list not included in the Bedford College list are social withdrawal and pathological guilt, whereas a key symptom in the latter list is preoccupational brooding). The Bedford College criterion also defines high-, medium- and low-borderline depressive conditions. The medium-borderline condition specified for the classification of chronic subclinical conditions requires depressed mood and two of the key symptoms. Those with high-borderline case conditions (with three key symptoms) are excluded from the chronic subclinical conditions category to maximise clarity in rating a new onset later. Low-borderline case depressions are not classified as being chronic subclinical conditions nor are low-borderline case anxieties (only one phobic object).

The Self Evaluation and Social Support Schedule (SESS) ${ }^{41}$ was used to rate the second component of the vulnerability index. Negative evaluation of core relationships involves a 'marked' or 'moderate' rating on a four-point scale of 'negative interaction' with a child living at home or, if married, in relation to her husband or partner. Both take into account reports about arguing, strain, violence and indifference, and ignoring anything positive about a relationship. Single women are also included as scoring positively on the negative evaluation of core relationships if they have negative interaction with another close relative or household member, or if they lack regular confiding contact with someone they define as very close.

Negative evaluation of self, the third component of the vulnerability index, involves low self-esteem as defined by a score of 'marked' or 'moderate' on any of the three four-point scales dealing with negative comments about (a) personal attributes, such as intelligence, attractiveness and ability to get on with people, (b) competence in roles, such as wife, mother, worker, and (c) lack of self acceptance - more generalised feelings about the way someone sees herself. In the Islington sample it was measured at the time of first interview and was found to be predictive of onset of depression. ${ }^{38}$

The Life Events and Difficulties Schedule (LEDS) employs a semi-structured interview and is based on a system of contextual measures reflecting the likely meaning of events and difficulties. ${ }^{42,43}$ These are contrasted with self-report or subjective ratings that record what the respondent actually felt about the event - for example, how upsetting she found it. The date of each event is recorded in terms of week of occurrence. Severe events are defined as having a severe long-term threat 10-14 days after their occurrence based on a judgement that takes into account relevant biographical and current circumstances, but ignores any report of emotional response. The ratings are referred to as 'contextual' because this procedure encompasses a much broader range of material than mere details of the event itself. Onset of depression has been linked with severe events occurring in various time periods, ${ }^{42}$ but there is general agreement that almost all of aetiological importance probably occur within 6 months of onset - usually within a matter of weeks. ${ }^{42}$ Severe 
long-term difficulties are ongoing problems such as cramped housing or poor relationships that may not necessarily give rise to events as defined but are rated on parallel scales of severity if lasting 4 weeks or more. ${ }^{44} \mathrm{~A}$ combined index of 'new provoking agent' used here identifies experience of either a new severe difficulty or a severe event between baseline and follow-up.

\section{Saliva samples and cortisol assay}

Participants provided samples of saliva around $08.00 \mathrm{~h}$ and $20.00 \mathrm{~h}$ over 4-10 days by drooling into a labelled tube within a week of the interview. Each saliva sample was assayed separately. Mean levels of cortisol were derived from each set. Cortisol was measured by enzyme-linked immunosorbent assay (ELISA) on $20 \mu \mathrm{l}$ samples of saliva without extraction (antibody Cambio UK). Intra-assay variation was $5.1-5.7 \%$ and inter-assay variation $5.6-6.7 \%$ for each set of samples. Details of the assay are given elsewhere. ${ }^{33}$ Levels are reported in $\mathrm{ng} / \mathrm{ml}$. Stages of the menstrual cycle were not recorded, but were likely to be evenly distributed in a sample of this size. There is no evidence that cortisol varies predictably during the menstrual cycle. ${ }^{45}$ Moreover in sample 1 no differences had emerged according to the stage of menstrual cycle. $^{33}$

\section{Genotyping}

We harvested DNA from a separate sample of saliva (Oragene, Canada), and genotyped for the Val/66/Met variant in rs6265 of the $B D N F$ gene by a commercial laboratory (Source Bioscience, UK).

\section{Follow-up}

Participants were contacted by telephone at 3-4 monthly intervals for about 12 months to monitor onset of depression or the occurrence of life events. If onset had occurred, the final follow-up interview took place in the participant's home soon afterwards. Otherwise, the final interview was carried out 12-14 months after the baseline interview. At this time crucial measures of the SCAN and LEDS were repeated for the whole of the intervening period.

\section{Statistical analysis}

We used binary regression to predict the risk of onset of a major depressive episode during follow-up. There were no cases of bipolar disorder. Mean morning cortisol, stressful life events and $B D N F$ genotype were the predictor variables of interest. Their direct effect and two-way interactions were tested as significant risk difference in binomial regression models, as previously recommended. ${ }^{46}$

Mean morning cortisol was cleaned to remove outlier measurements and adjusted for time of sampling before being used as a predictor in the binary regressions (see below). Since previous evidence suggested a U-shaped relationship between cortisol and risk of depression, ${ }^{3}$ we have systematically tested both linear and quadratic (curved) effects.

\section{Results}

Of the 279 women vulnerable to depression and willing to participate, $58 \%$ had a negative evaluation of self, $52 \%$ chronic subclinical conditions and $33 \%$ negative elements in core relationships. The average age was 36.8 years.

\section{Baseline salivary morning cortisol}

We first examined the distribution of the baseline morning cortisol values. Cortisol data were available for 279 women from the three samples. In sample 1, cortisol levels were measured on 4 consecutive days in 44 women (average number of valid measurements: 3.9). In samples 2 and 3, cortisol was measured on 10 consecutive days; 155 women provided on average 9.3 valid cortisol values. In sample 3, 80 women provided on average 9.1 valid levels. Across the three samples, a total of 2346 cortisol measurements were available for the 279 women. To minimise the effect of measurement inaccuracies and random factors affecting the cortisol measures, we first checked the measured cortisol values for outliers. Of the 2346 measurements, 15 were two standard deviations or more below and 47 were two standard deviations or more above the mean value of the remaining measurements. These 62 measurements were considered outliers.

The remaining 2284 measurements were considered valid and used for calculating individual means. Cortisol levels did not vary with measurement day $(1-0)(b=-0.004,95 \%$ CI -0.025 to $0.017, P=0.74)$. However, cortisol levels decreased significantly with time of the day, approximately $0.38 \mathrm{ng} / \mathrm{ml}$ every hour ( $b=-0.390,95 \% \mathrm{CI}-0.505$ to $-0.275, P<0.0001$ ). Therefore, we adjusted cortisol measurements for sampling time (using first and second polynomial of time). The mean values for each individual were calculated as arithmetic means of on average 8.2 (range 3-10) measurements adjusted for sampling time. Adjusted cortisol levels did not differ by sample $(F(2,276)=2.25, P=0.11)$ and were nearly normally distributed with a slight right skew (Fig. 1).

\section{Onset of depression}

There were 53 onsets of major depressive episodes (19\% of the total sample) during the study. Analysis of the three samples individually showed that while sample 1 and 2 showed similar incidences of onset (29.5\% and $23.4 \%$ respectively), this was lower in sample $3(9.7 \%)\left(\chi^{2}=7.22, P=0.027\right)$. Therefore, the study sample was controlled for in all subsequent analyses. Onset was unrelated to experience of a previous depressive episode or to baseline chronic subclinical symptoms of either depression or other conditions. Neither negative elements in core relationships nor negative evaluation of self predicted the subsequent onset of major depression in this data-set (logistic regression: $P>0.05$ in each case). Nor was there any association between morning

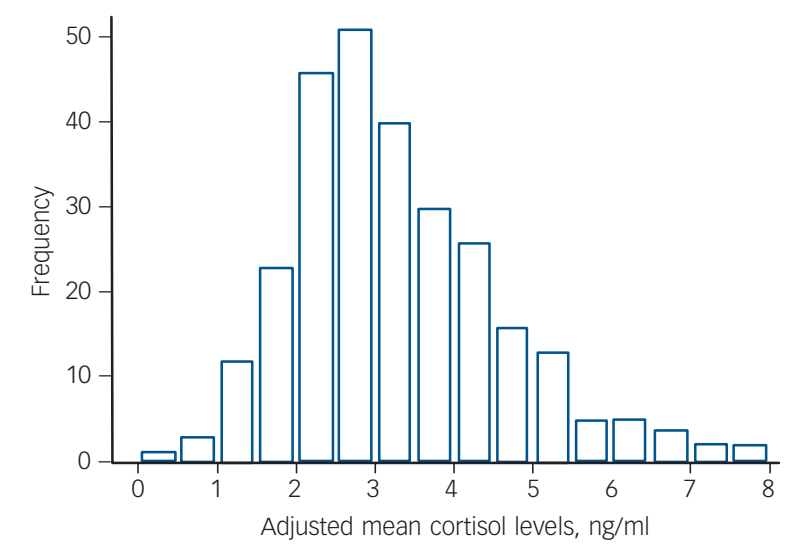

Fig. 1 Distribution of average morning cortisol levels, adjusted for sampling time. 
cortisol and any of the three vulnerability factors (chronic subclinical symptoms, negative elements in core relationships, negative evaluation of self). Association between a score conveying the number of such factors experienced by each woman and depressive onset, although clear among the whole data-set $(P=0.038)$, just failed to reach statistical significance among those participants with data on both cortisol and BDNF.

\section{Morning cortisol levels and the onset of depression}

Next, we asked whether individual differences in cortisol values predicted the subsequent onset of an episode of major depression. There was a significant U-shaped relationship between adjusted morning cortisol levels at baseline and the probability of depression onset during follow-up. This is shown in Fig 2. This relationship was controlled for sample (i.e. sample 1, 2 or 3 ) as a covariate. The risk difference per $\mathrm{ng} / \mathrm{ml}$ cortisol was 0.028 (95\% CI $0.011-0.045, P=0.001)$. The odds ratio was $1.12(95 \%$ CI $1.01-1.21, P=0.031)$. There was no significant interaction between sample and mean adjusted morning cortisol in predicting onset $(P>0.1)$.

\section{New provoking agent, cortisol and onset of depression}

Many studies have shown that an adverse life event precedes major depression, and we confirmed that this was the case in this study. In total, $51 \%$ of the total sample experienced at least one severe life event during follow-up. As expected, such an experience strongly predicted subsequent onset of depression (risk difference: $0.254,95 \%$ CI $0.159-0.349, P<0.001)$. The occurrence of a severe life event was independent of baseline levels of morning cortisol. Adjusted mean cortisol levels did not significantly predict the subsequent occurrence of a severe life event either linearly $(P=0.1)$ or as a curved relationship $(P=0.1)$.

We then went on to examine whether such agents and cortisol interacted to predict depression. There was no significant interaction between the curved cortisol function (quadratic) and a severe life event in predicting depressive onsets (risk difference: $-0.00092,95 \%$ CI -0.01001 to $0.0082, P=0.842$ ), whereas severe life events and the mean adjusted baseline cortisol remained significant predictors of depressive onset (Table 1).

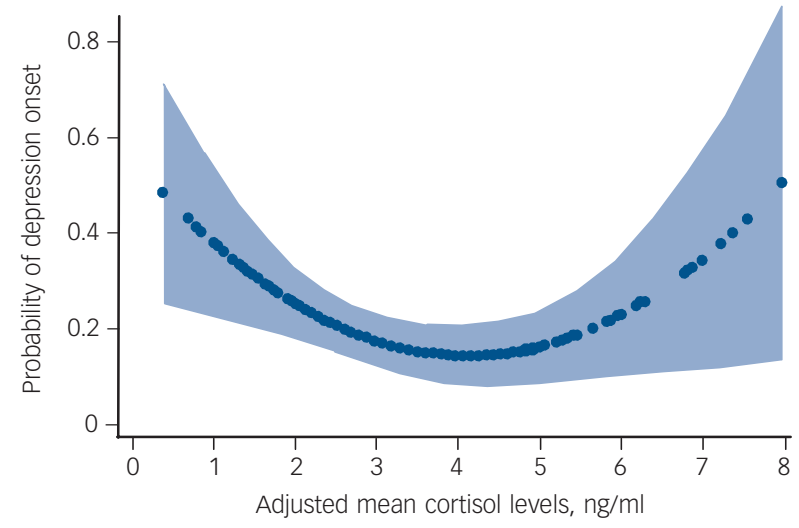

Fig. 2 The relationship between cortisol level at baseline and the risk of onset of a new depressive episode during the

follow-up based on the best fitting model estimates. The light blue shading is the $95 \%$ confidence interval of these estimates.

\section{Interaction between morning cortisol levels and BDNF Val/66/Met in predicting onset of depression}

We next examined the contribution of the common BDNF Val/66/ Met polymorphism as a predictor of the onset of an episode of major depression. The BDNF rs6265 (Val/66/Met) genotypes in this study were distributed as expected under Hardy-Weinberg equilibrium $\left(\chi^{2}=0.157, P=0.69\right)$, with a minor allele frequency of 0.20 . For subsequent analyses, we coded rs6265 under the dominant Met allele genetic model, contrasting 88 Met allele carriers with $157 \mathrm{Val}$ allele homozygotes. Table 1 sets out these analyses in detail.

The BDNF Val/66/Met genotype was not directly associated with onsets of depression (risk difference: 0.049, 95\% CI -0.067 to $0.164, P=0.229$ ) in this data-set, or with any of the three vulnerability factors or their composite score. However, there was a significant interaction between the quadratic cortisol function and the BDNF Val/66/Met genotype in their effect on depressive onsets under the additive statistical model (interaction risk difference: $-0.010,95 \%$ CI -0.022 to $-0.0001, P=0.048$ ) (Table 1).

\begin{tabular}{|c|c|c|c|}
\hline & Risk difference $(95 \% \mathrm{Cl})$ & z-score & $P$ \\
\hline \multicolumn{4}{|c|}{ Interaction between mean adjusted $08.00 \mathrm{~h}$ cortisol and stressful life events ${ }^{\mathrm{a}}$} \\
\hline Severe stressful life events & $0.168(0.099$ to 0.237$)$ & 4.79 & 0.001 \\
\hline Cortisol: linear effect & $-0.164(-0.315$ to -0.0137$)$ & -2.14 & 0.032 \\
\hline Cortisol: quadratic effect & 0.019 (0.000 to 0.039$)$ & 1.96 & 0.049 \\
\hline Interaction & $-0.001(-0.010$ to 0.008$)$ & -0.20 & 0.842 \\
\hline \multicolumn{4}{|c|}{ Interaction between cortisol and BDNF genotype (binary: presence/absence of Met allele) ${ }^{\mathrm{b}}$} \\
\hline Cortisol: linear effect & $-0.289(-0.464$ to -0.115$)$ & -3.25 & 0.001 \\
\hline Cortisol: quadratic effect & $0.033(0.0108$ to 0.055$)$ & 2.92 & 0.004 \\
\hline$B D N F$ & $0.031(-0.075$ to 0.137$)$ & 0.57 & 0.565 \\
\hline Interaction (quadratic) & $-0.010(-0.021$ to 0.000$)$ & -1.98 & 0.048 \\
\hline \multicolumn{4}{|c|}{ Analyses stratified by BDNF genotypes ${ }^{c}$} \\
\hline \multicolumn{4}{|l|}{ Val/Val homozygotes } \\
\hline Cortisol: linear effect & $-0.315(-0.553$ to -0.079$)$ & -2.61 & 0.009 \\
\hline Cortisol: quadratic effect & $0.042(0.010$ to 0.072$)$ & 2.62 & 0.009 \\
\hline \multicolumn{4}{|l|}{ Met carriers } \\
\hline Cortisol: linear effect & $-0.267(-0.524$ to -0.009$)$ & -2.03 & 0.042 \\
\hline Cortisol: quadratic effect & $0.025(-0.006$ to 0.056$)$ & 1.57 & 0.115 \\
\hline
\end{tabular}


Further examination of these results showed that the $\mathrm{U}$-shaped relationship between the mean adjusted cortisol at baseline and depressive onsets during the follow-up period was limited to the Val/Val homozygotes but was not apparent in the Met allele carriers. This is shown in Fig. 3 and Table 1. In Met allele carriers, there was a weak (non-significant) negative linear relationship with higher cortisol values being protective against depression.

There was no significant interaction between BDNF Val/66/ Met and severe life events in predicting subsequent onset of depression in this data-set (interaction risk difference: -0.139 , 95\% CI -0.068 to $0.345, P=0.187$ ).

\section{Discussion}

\section{Main findings}

Previous studies have shown that increasing levels of morning cortisol, as measured in the saliva, are associated with increased risk of depression. ${ }^{33,34}$ The results reported in this paper suggest, in addition, that those with unusually low morning cortisol also showed such an association; there was a quadratic relationship between the hormone and the disorder. The occurrence of an adverse life event is also a major predictor, but neither in this study nor in previous ones on adolescents and adult women did we find any association between salivary cortisol levels and life events as interacting predictors of depression. ${ }^{33,34}$ However, it should be noted that there is no information on the ways that cortisol might alter in response to such life events, and hence play a more proximal role in the likelihood of consequent depression. We did not measure dehydroepiandrosterone, since our previous

(a)

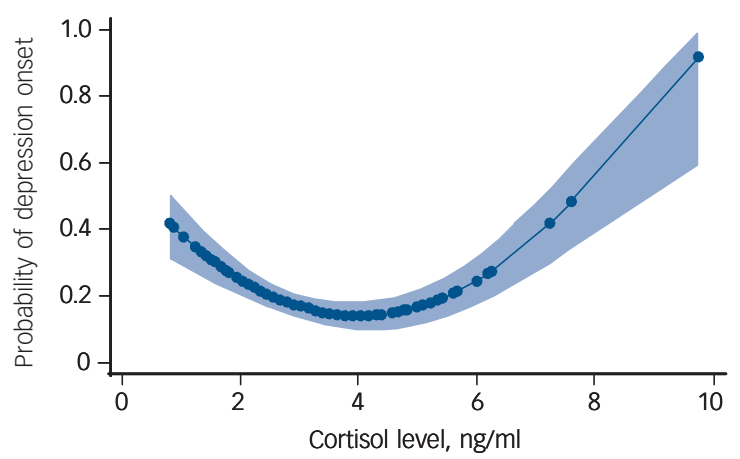

(b)

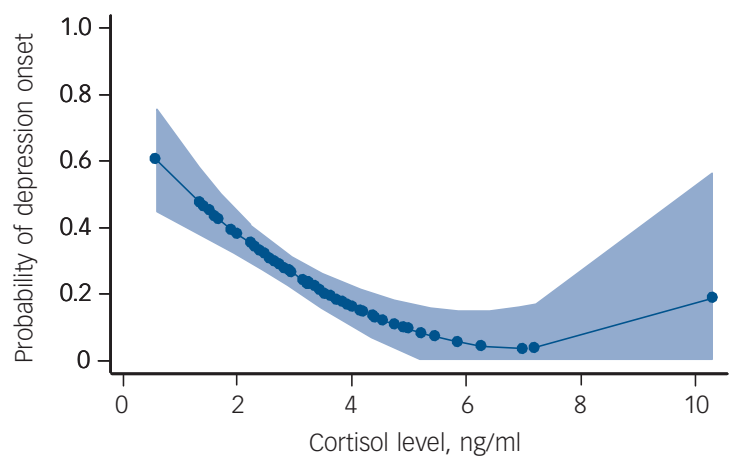

Fig. 3 The relationship between cortisol level at baseline and the risk of onset of a new depressive episode during the follow-up separately for BDNF rs6265 Val allele homozygotes (a) and for Met allele carriers (b), based on the best fitting model estimates. The light blue shading is the $95 \%$ confidence interval of these estimates studies have failed to show that this steroid is a risk factor for major depression on its own, or that it interacts with BDNF. ${ }^{34,35,47}$

We found that the predictive value of morning salivary cortisol depends on the BDNF rs6265 variant. In agreement with our previous study on adolescents (in which we tested for a linear function), higher levels of morning cortisol appear to represent a risk factor for depression only in those carrying the BDNF Val/66/Val variant. ${ }^{35}$ This replication strengthens the interpretation that this association is a generalised risk factor, for there are important differences between the two studies. Not only were the adolescents younger than the participants studied here, but their depressive episode was a first onset, whereas in this study, many of the participants had had previous depressive episodes. There have been a number of suggestions that antecedent factors for first onset and recurrent episodes of depression might be different. ${ }^{48}$ There were insufficient participants in this study for us to determine whether the $\mathrm{BDNF} /$ cortisol interaction we describe applied both to those with first and/or recurrent onsets.

Our findings, together with a previous report, ${ }^{35}$ suggest that the BDNF Val/66/Met moderates the risk for major depression represented by higher cortisol. It should be noted that we found a quadratic relation between depressive onset and cortisol: as well as higher cortisol predicting increased risk for depression in these participants, there was some evidence that levels in the lower range also represented increased risk. This needs to be confirmed in a larger study, but it would not be unusual for hormones to operate in an optimal range, and for departures in either direction to have adverse consequences. It should also be noted that previous studies ${ }^{20,25,26}$ (see introduction) have related the Met $B D N F$ allele to increased risk for major depression in some contexts. However, it is plausible that one allele of a common genetic variant may moderate one set of risk factors (for example, psychosocial, such as early adversity in childhood or following severe life events in adulthood ${ }^{23,26,27}$ ) but another allele may do so in the context of a different one (for example levels of hormones such as cortisol in adulthood). Differing vulnerabilities according to circumstance have also been postulated for the common $s / l$ variant in the serotonin transporter, so that a given polymorphism may be disadvantageous in one context, but beneficial in another. ${ }^{49}$

\section{Limitations}

It is important to recognise that our study population was not a single one, but made up of three separate samples collected from different geographical areas (although within the same country) and at different times (in one case). The size of each sample was not sufficient for us to test whether the interaction we report on the total sample might apply to each one individually. Nevertheless, the fact that each was collected according to similar criteria bolsters our contention that they can be considered together. The only marked difference was that in one sample (Oxford) the incidence of life events (and depression) was lower than in the other two. Since we found no interaction between the occurrence of life events and the BDNF variant when studied as combined predictors of subsequent depression, we think that this is not a significant problem with our data. However, to confirm that results are not driven by any unmeasured differences, we corrected for sample in all analyses.

\section{The nature of the interaction}

In studies that report an interaction between a genetic variation and some physiological parameter (for example a steroid hormone) in predicting a presumed dependent outcome (in this 
case, depression), it is always important to exclude the possibility that the genetic variant is associated with (and, maybe, responsible for) individual differences in the measured parameter, in this case cortisol. Since in this study, as in our previous one, ${ }^{35}$ we found no direct association between BDNF rs6265 and individual values in morning cortisol, we can exclude this possibility. It therefore seems feasible that this variation in the BDNF gene and cortisol may interact at a neural level to influence the risk for depression.

There is no direct neural evidence for such an interaction in humans, largely because current techniques do not allow it to be studied. But there is plentiful evidence from experimental studies for an interaction between BDNF and glucocorticoids. For example, excess corticosterone decreases the expression of BDNF mRNA in the hippocampus. ${ }^{9,50}$ Excess corticoid also interferes with the stimulating action of fluoxetine on hippocampal neurogenesis, ${ }^{51}$ although, as we point out earlier, the link between hippocampal neurogenesis and depression is still uncertain. Reduced hippocampal volumes have been reported both in participants with current depression and those at high risk of depression, although whether this relates directly to impaired neurogenesis is still undetermined. ${ }^{52}$ Brain-derived neurotrophic factor has a well-established role in promoting neural plasticity, and this has been related both to the onset of depression and to the therapeutic response to antidepressant drugs. ${ }^{1,2,53}$ In this context, it is interesting that there is now experimental evidence that fluoxetine also promotes neural plasticity, ${ }^{54}$ although whether this is modulated by corticoids has not yet been reported. Since corticoids reduce BDNF mRNA, it is reasonable to conclude that the effect of BDNF on plasticity would also be reduced by higher levels of cortisol. The BDNF Val/66/Met variant does not occur naturally in rodents, although it can be produced artificially. It will be interesting to see whether there are experimental interactions between this variant and glucocorticoids that assist the interpretation of the results we present in this paper.

In conclusion, the emerging consensus that the role of particular genetic variants as risk factors for depression are best studied as interactions with either environmental events or physiological variables ('endophenotypes') is supported by the data presented here. This paper reaffirms that individual differences in cortisol, known to be a risk factor for depression, are moderated by common variants in $B D N F$ as well as other genes.

J. Herbert, MB ChB, PhD, Cambridge Centre for Brain Repair, Department of Clinical Neurosciences, University of Cambridge, Cambridge, UK; M. Ban, PhD, Department of Neurology, Department of Clinical Neurosciences, University of Cambridge, Cambridge, UK; G. W. Brown, PhD, T. O. Harris, DHC, Department of Health Service and Population Research, Institute of Psychiatry, London, UK; A. Ogilvie, MB ChB, MRCPsych, Department of Psychiatry, University of Oxford, Oxford, UK; R. Uher, MD, PhD, MRCPsych, Social, Genetic, and Developmental Psychiatry Research Centre, King's College London, UK and currently Department of Psychiatry, Dalhousie University, Halifax, Canada; T. K. J. Craig, MBBS, PhD, FRCPsych, Department of Health Service and Population Research, Institute of Psychiatry, London, UK

Correspondence: J. Herbert, Cambridge Centre for Brain Repair, Department of Clinical Neurosciences, University of Cambridge, Cambridge CB2 OSP, UK. Email: jh24@cam.ac.uk

First received 6 Dec 2011, final revision 24 May 2012, accepted 14 June 2012

\section{Funding}

These studies were supported by grants from the Medical Research Council and the Wellcome Trust (grant number: 066693/Z/01/Z).

\section{Acknowledgements}

We would like to thank the general practitioners who collaborated in this study, as well as Sophie Borsanyi, Sofia Messari, Katherine Stanford, Kirsty Bowen and Amy Mclachlan who helped collect the data. We are grateful to Helen Shiers and Sarah Cleary for the cortisol assays. Finally, we would like to thank all those women who were willing to give their time to be interviewed and to collect their saliva for the project.

\section{References}

1 Duman RS, Monteggia LM. A neurotrophic model for stress-related mood disorders. Biol Psychiatry 2006; 59: 1116-27.

2 D'Sa C, Duman RS. Antidepressants and neuroplasticity. Bipolar Disord 2002; 4: $183-94$.

3 Garcia R. Stress, metaplasticity, and antidepressants. Curr Mol Med 2002; 2: 629-38.

4 Alme MN, Wibrand K, Dagestad G, Bramham CR. Chronic fluoxetine treatment induces brain region-specific upregulation of genes associated with BDNF-induced long-term potentiation. Neural Plast 2007; 2007: 264-96.

5 De Foubert G, Carney SL, Robinson CS, Destexhe EJ, Tomlinson R, Hicks CA, et al. Fluoxetine-induced change in rat brain expression of brain-derived neurotrophic factor varies depending on length of treatment. Neuroscience 2004; 128: 597-604.

6 Pinnock SB, Lazic SE, Wong HT, Wong $\mathrm{IH}$, Herbert J. Synergistic effects of dehydroepiandrosterone and fluoxetine on proliferation of progenitor cells in the dentate gyrus of the adult male rat. Neuroscience 2009; 158: 1644-51.

7 Rantamaki T, Hendolin P, Kankaanpaa A, Mijatovic J, Piepponen P, Domenici $\mathrm{E}$, et al. Pharmacologically diverse antidepressants rapidly activate brain-derived neurotrophic factor receptor TrkB and induce phospholipasecgamma signaling pathways in mouse brain. Neuropsychopharmacology 2007; 32: 2152-62.

8 Schmidt HD, Duman RS. The role of neurotrophic factors in adult hippocampal neurogenesis, antidepressant treatments and animal models of depressive-like behavior. Behav Pharmacol 2007: 18: 391-418.

9 Pinnock SB, Blake AM, Platt NJ, Herbert J. The roles of BDNF, pCREB and Wnt3a in the latent period preceding activation of progenitor cell mitosis in the adult dentate gyrus by fluoxetine. PLOS One 2010; 5: e13652.

10 Pinnock SB, Herbert J. Brain-derived neurotropic factor and neurogenesis in the adult rat dentate gyrus: interactions with corticosterone. Eur J Neurosci 2008; 27: 2493-500.

11 Malberg JE, Duman RS. Cell proliferation in adult hippocampus is decreased by inescapable stress: reversal by fluoxetine treatment Neuropsychopharmacology 2003; 28: 1562-71.

12 Malberg JE, Eisch AJ, Nestler EJ, Duman RS. Chronic antidepressant treatment increases neurogenesis in adult rat hippocampus. $J$ Neurosci 2000; 20: 9104-10.

13 Karege F, Perret G, Bondolfi G, Schwald M, Bertschy G, Aubry JM. Decreased serum brain-derived neurotrophic factor levels in major depressed patients. Psychiatry Res 2002; 109: 143-8.

14 Karege F, Bondolfi G, Gervasoni N, Schwald M, Aubry JM, Bertschy G. Low brain-derived neurotrophic factor (BDNF) levels in serum of depressed patients probably results from lowered platelet BDNF release unrelated to platelet reactivity. Biol Psychiatry 2005; 57: 1068-72.

15 Lee BH, Kim H, Park SH, Kim YK. Decreased plasma BDNF level in depressive patients. J Affect Disord 2007; 101: 239-44.

16 Chen ZY, Patel PD, Sant G, Meng CX, Teng KK, Hempstead BL, et al. Variant brain-derived neurotrophic factor (BDNF) (Met66) alters the intracellular trafficking and activity-dependent secretion of wild-type BDNF in neurosecretory cells and cortical neurons. J Neurosci 2004; 24: 4401-11.

17 Schumacher J, Jamra RA, Becker T, Ohlraun S, Klopp N, Binder EB, et al. Evidence for a relationship between genetic variants at the brain-derived neurotrophic factor (BDNF) locus and major depression. Biol Psychiatry 2005; 58: $307-14$

18 Levinson DF. The genetics of depression: a review. Biol Psychiatry 2006; 60 84-92.

19 Hong CJ, Huo SJ, Yen FC, Tung CL, Pan GM, Tsai SJ. Association study of a brain-derived neurotrophic-factor genetic polymorphism and mood disorders, age of onset and suicidal behavior. Neuropsychobiology 2003; 48: 186-9.

20 Caspi A, Sugden K, Moffitt TE, Taylor A, Craig IW, Harrington H, et al. Influence of life stress on depression: moderation by a polymorphism in the 5-HTT gene. Science 2003; 301: 386-9.

21 Bukh JD, Bock C, Vinberg M, Werge T, Gether U, Kessing LV. No interactions between genetic polymorphisms and stressful life events on outcome of antidepressant treatment. European Neuropsychopharmacol 2010; 20: 327-35.

22 Karg K, Burmeister M, Shedden K, Sen S. The serotonin transporter promoter variant (5-HTTLPR), stress, and depression meta-analysis revisited: evidence of genetic moderation. Arch Gen Psychiatry 2011; 68: 444-54. 
23 Kim JM, Stewart R, Kim SW, Yang SJ, Shin IS, Kim YH, et al. Interactions between life stressors and susceptibility genes (5-HTTLPR and BDNF) on depression in Korean elders. Biol Psychiatry 2007; 62: 423-8.

24 Uher R, McGuffin P. The moderation by the serotonin transporter gene of environmental adversity in the etiology of depression: 2009 update. Mol Psychiatry 2010; 15: 18-22.

25 Kim JM, Stewart R, Kim SW, Yang SJ, Shin IS, Kim YH, et al. BDNF genotype potentially modifying the association between incident stroke and depression. Neurobiol Aging 2008; 29: 789-92.

26 Bukh JD, Bock C, Vinberg M, Werge T, Gether U, Vedel Kessing L. Interaction between genetic polymorphisms and stressful life events in first episode depression. J Affect Disord 2009; 119: 107-15.

27 Kaufman J, Yang BZ, Douglas-Palumberi H, Grasso D, Lipschitz D, Houshyar S, et al. Brain-derived neurotrophic factor-5-HTTLPR gene interactions and environmental modifiers of depression in children. Biol Psychiatry 2006; 59 673-80.

28 Carver CS, Johnson SL, Joormann J, Lemoult J, Cuccaro ML. Childhood adversity interacts separately with 5 -HTTLPR and BDNF to predict lifetime depression diagnosis. J Affect Disord 2011; 132: 89-93.

29 Aguilera M, Arias B, Wichers M, Barrantes-Vidal N, Moya J, Villa H, et al. Early adversity and 5-HTT/BDNF genes: new evidence of gene-environment interactions on depressive symptoms in a general population. Psychol Med 2009; 39: 1425-32.

30 Sachar EJ, Hellman L, Roffwarg HP, Halpern FS, Fukushima DK, Gallagher TF. Disrupted 24-hour patterns of cortisol secretion in psychotic depression. Arch Gen Psychiatry 1973; 28: 19-24.

31 Carroll BJ. Use of the dexamethasone suppression test in depression. J Clin Psychiatry 1982; 43: 44-50.

32 Rush AJ, Giles DE, Schlesser MA, Orsulak PJ, Parker Jr CR, Weissenburger JE, et al. The dexamethasone suppression test in patients with mood disorders. J Clin Psychiatry 1996; 57: 470-84.

33 Harris TO, Borsanyi S, Messari S, Stanford K, Brown GW, Cleary SE, et al Morning cortisol as a risk factor for subsequent major depressive disorder in adult women. Br J Psychiatry 2000; 177: 505-10.

34 Goodyer IM, Tamplin A, Herbert J, Altham PM. Recent life events, cortisol, dehydroepiandrosterone and the onset of major depression in high-risk adolescents. Br J Psychiatry 2000; 177: 499-504.

35 Goodyer IM, Croudace T, Dudbridge F, Ban M, Herbert J. Polymorphisms in BDNF (Val66Met) and 5-HTTLPR, morning cortisol and subsequent depression in at-risk adolescents. Br J Psychiatry 2010; 197: 365-71.

36 Wing JK, Barbor T, Brugha TS. SCAN: schedule for clinical assessment in neuropsychiatry. Arch Gen Psychiatry 1990; 47: 589-93.

37 Brown GW, Bifulco A, Harris T, Bridge L. Life stress, chronic subclinical symptoms and vulnerability to clinical depression. J Affect Disord 1986; 11 $1-19$.
38 Brown GW, Andrews B, Harris T, Adler Z, Bridge L. Social support, selfesteem and depression. Psychol Med 1986; 16: 813-31.

39 American Psychiatric Association. Diagnostic and Statistical Manual of Mental Disorders (4th edn) (DSM-IV). APA, 1994

40 Finlay-Jones R, Brown GW, Duncan-Jones P, Harris T, Murphy E, Prudo R. Depression and anxiety in the community: replicating the diagnosis of a case. Psychol Med 1980; 10: 445-54.

41 Andrews B, Brown GW. Self Evaluation and Social Support (SESS) Manual. Royal Holloway College, 1991.

42 Brown GW, Harris TO. Social Origins of Depression: A Study of Psychiatric Disorder in Women. Tavistock Publications, 1978

43 Brown GW, Harris TO. Life Events \& Illness. Guilford Press, 1989

44 Brown GW, Bifulco A, Harris TO. Life events, vulnerability and onset of depression: some refinements. Br J Psychiatry 1987; 150: 30-42.

45 Nepomnaschy PA, Altman RM, Watterson R, Co C, McConnell DS, England BG. Is cortisol excretion independent of menstrual cycle day? A longitudinal evaluation of first morning urinary specimens. PLOS One 2011; 6: e18242.

46 Rothman KJ, Greenland RW, Lash TL. Modern Epidemiology. Lippincott Williams \& Wilkins, 2008.

47 Young $\mathrm{AH}$, Gallagher P, Porter RJ. Elevation of the cortisoldehydroepiandrosterone ratio in drug-free depressed patients. $\mathrm{Am}$ Psychiatry 2002; 159: 1237-9.

48 Kendler KS, Thornton LM, Gardner CO. Genetic risk, number of previous depressive episodes, and stressful life events in predicting onset of major depression. Am J Psychiatry 2001; 158: 582-6.

49 Homberg JR, Lesch KP. Looking on the bright side of serotonin transporter gene variation. Biol Psychiatry 2011; 69: 513-9.

50 Pinnock SB, Balendra R, Chan M, Hunt LT, Turner-Stokes T, Herbert J. Interactions between nitric oxide and corticosterone in the regulation of progenitor cell proliferation in the dentate gyrus of the adult rat. Neuropsychopharmacology 2007; 32: 493-504.

51 Huang GJ, Herbert J. Stimulation of neurogenesis in the hippocampus of the adult rat by fluoxetine requires rhythmic change in corticosterone. Biol Psychiatry 2006; 59: 619-24.

52 Frodl T, Reinhold E, Koutsouleris N, Reiser M, Meisenzahl EM. Interaction of childhood stress with hippocampus and prefrontal cortex volume reduction in major depression. J Psychiatr Res 2010; 44: 799-807.

53 Castren E, Rantamaki T. Neurotrophins in depression and antidepressant effects. Novartis Found Symp 2008; 289: 43-52; discussion 3-9, 87-93.

54 Maya Vetencourt JF, Sale A, Viegi A, Baroncelli L, De Pasquale R, O'Leary OF, et al. The antidepressant fluoxetine restores plasticity in the adult visual cortex. Science 2008; 320: 385-8. 